Volume 3

June 2017

\title{
Processus de Démocratisation et Polarisation d'une Société. Une Analyse de la Crise Actuelle au Burundi (Avril 2015-Juin 2016)
}

Leonidas Ndayisaba

University of Burundi, leonidasndayisab@hotmail.com

Follow this and additional works at: https://digitalcommons.usf.edu/jacaps

Part of the Social and Behavioral Sciences Commons

\section{Recommended Citation}

Ndayisaba, Leonidas (2017) "Processus de Démocratisation et Polarisation d'une Société. Une Analyse de la Crise Actuelle au Burundi (Avril 2015-Juin 2016)," Journal of African Conflicts and Peace Studies: Vol. 3: Iss. 2, 38-50.

DOI: https://doi.org/10.5038/2325-484X.3.2.1100

Available at: https://digitalcommons.usf.edu/jacaps/vol3/iss2/3

This Article is brought to you for free and open access by the Open Access Journals at Digital Commons @ University of South Florida. It has been accepted for inclusion in Journal of African Conflicts and Peace Studies by an authorized editor of Digital Commons @ University of South Florida. For more information, please contact digitalcommons@usf.edu. 


\section{Introduction}

Depuis l'année 2015, l'Afrique centrale entre dans une crise politique et institutionnelle due aux termes de la constitution, en ce qu'elle limite le mandat présidentiel à cinq ans pour la plupart des Etats, renouvelable une seule fois, donc dix ans au maximum. Cette décision, quoique réglée dans la constitution de ces différents pays, constitue actuellement un facteur de polarisation au sein de la société et de la classe politique. 14 pays africains organisent cette année des élections générales : le Congo (Brazzaville) a déjà modifié la constitution pour que l'actuel président puisse se faire élire cette année ; les élections apparaissent incertaines cette année en République Démocratique du Congo ${ }^{1}$. Au Burundi, la décision de l'actuel Chef de l'Etat, Pierre Nkurunziza, annoncée en avril 2015, de se porter candidat pour un troisième mandat, a plongé le pays dans une crise institutionnelle et ses conséquences se font sentir à plus d'un égard. Partisans et opposants à cette nouvelle candidature trouvent certes leurs arguments respectifs dans les mêmes sources politiques et légales, mais avec des interprétations diamétralement opposées.

Ceci dit, des élections générales ont été organisées en 2015, du niveau local au niveau national, permettant à l'exécutif de renforcer son pouvoir. Certes, le pouvoir et les forces de sécurité semblent maîtriser la situation dans un rapport de forces pour le moment asymétrique, mais, le pays se trouve à la croisée des chemins tant la crise s'avère multidimensionnelle avec des conséquences imprévisibles pour le pays et l'ensemble de la région. Ainsi, la Communauté est-africaine (CEA) et les partenaires internationaux du Burundi s'emploient actuellement à encourager les parties internes et externes à un dialogue inclusif.

Cette contribution a pour objectif d'analyser la crise actuelle en cours au Burundi, dans ses dimensions politique, juridique, économique et sociale. Dans cet ordre d'idées, il sied de revisiter certaines dispositions de l'Accord d'Arusha pour la Paix et la Réconciliation au Burundi d'août 2000 dans sa lettre et son esprit, la Constitution du 28 mars 2005 qui s'en est inspirée. De surcroît, l'on ne saurait faire l'impasse sur certains aspects pertinents de la gestion du pouvoir pendant les dix dernières années. Des lois, des institutions étatiques existent, des programmes et projets de consolidation de la paix ont été conçus et mis en œuvre pendant cette période en vue de renforcer l'Etat et la société après des décennies d'instabilité politique et de guerre civile.

Pourtant la crise s'appuie également sur des décennies d'exercice du pouvoir, depuis les années quatre-vingt, qui n'ont pas pu répondre aux besoins collectifs de la population en dépit des avancées majeures du pays sur le plan politique : Chômage des jeunes, démographie galopante, urbanisation, absence de perspectives, autant de facteurs qui s'expriment également à travers la contestation contre le troisième mandat.

\footnotetext{
${ }^{1}$ ISS Weekly Press Release du 17 mars 2016, Infography
} 


\section{Carte du Burundi et de la Région des Grands Lacs Africains}

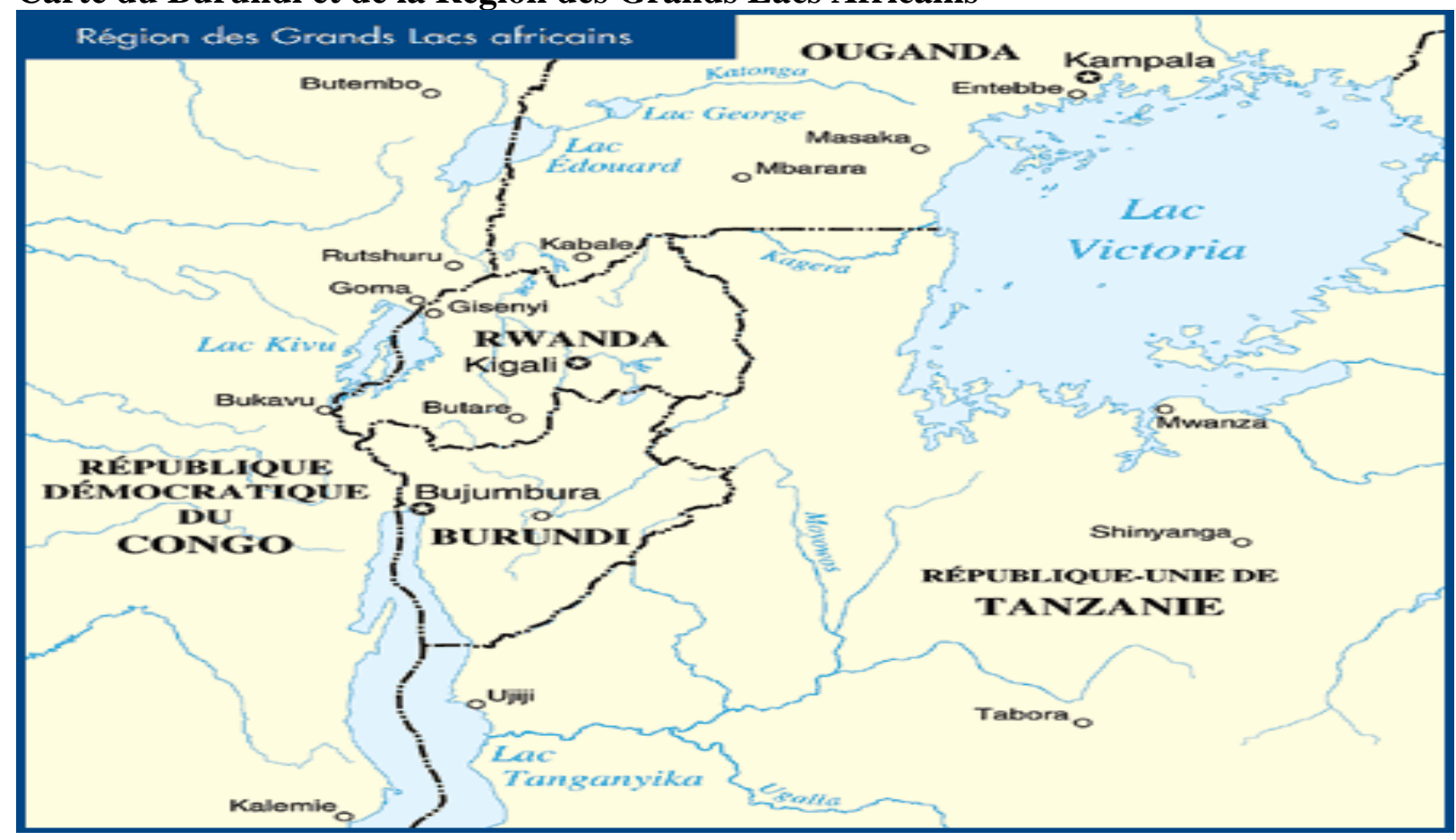

Source : www.alloprof.qc.ca, consulté le 14 juin 2016

\section{1) Une vielle nation confrontée à la gestion d'un jeune Etat indépendant}

Depuis son accession à l'indépendance le 1er juillet 1962, le Burundi connaît de façon récurrente une instabilité politique malgré l'héritage d'une constitution moderne adoptée le 16 avril $1962^{2}$. Cette dernière respecte à la fois la tradition monarchique du pays et la nécessité de créer des institutions étatiques modernes dans lesquelles tous les groupes peuvent se reconnaître. Ainsi, elle esquisse une monarchie constitutionnelle où «le Roi règne mais ne gouverne pas » tandis que le pouvoir est partagé entre les deux grands groupes dits ethniques, Hutu et Tutsi, dans les institutions tant au niveau national (gouvernement, parlement) qu'au niveau local (communes) $)^{3}$. La polarisation ethnique n'étant pas historique ni incorrigible, la question qui se pose est celle de savoir pourquoi elle revient de façon récurrente ${ }^{4}$. Il ne s'agit pas à cet effet de retracer l'historique du conflit mais plutôt d'évoquer seulement des points que nous jugeons pertinents de l'histoire du Burundi indépendant.

Cet héritage culturel, politique et constitutionnel n'aura pas été sauvegardé, les premières années du Burundi indépendant connaissent une instabilité politique : changement constat de l'équipe gouvernementale, des cas d'assassinats de hautes personnalités parfois des figures intégrationnistes comme le Héros de l'indépendance Louis Rwagasore en 1961, le Premier Ministre Pierre Ngendandumwe en 1965, rendant difficile le renouvellement des leaders responsables, mais permettant l'apparition de nouveaux clivages au sein de l'élite du pays

\footnotetext{
${ }^{2}$ Constitution définitive du Royaume du Burundi du 16 octobre 1962, BOB 1963, p.1; elle remplace la Constitution provisoire du Burundi du 28 novembre 1961, RJRB, 1962, p.62

${ }^{3}$ Voir Gahama Joseph / Stanislas Makoroka et. alii (1999) : Burundi ; in Adedeji Adebayo (ed.) : Comprehending and mastering African conflicts. The search for sustainable peace and good governance, London / New-York: Zed Books, pp.80-103

${ }^{4}$ Isabirye B. Stephen /Kooros Mahmoudi (2000) : Rwanda and Burundi : The dynamics of their " tribal conflicts ; in Africa Quarterly, Vol.40, Nr.4, pp.21-50;
} 
d'alors avec en arrière-fond l'appartenance «ethnique». L'avènement de la République en novembre 1966 marque la fin de la tradition monarchique du pays, de l'ordre constitutionnel établi avant l'accès à l'indépendance (1962-1966) et l'entrée du pays dans une nouvelle ère (1966-1992) jusqu'à l'avènement du processus démocratique.

Bref, la source de l'instabilité politique n'est pas le système politique esquissé par la constitution de 1962, mais plutôt l'incapacité des élites à gérer le pays ${ }^{5}$. Huit coups d'états militaires réussis ou échoués pour accéder au pouvoir et le contrôler ${ }^{6}$, des guerres civiles sur fonds de compétition politico-ethnique pour accéder et contrôler le pouvoir, des processus ratés de démocratisation d'abord au début des années 1960 et puis en 1991 et 1993 ont marqué l'histoire récente du pays. Cette compétition pour le pouvoir par les membres de l'élite Hutu et Tutsi s'accompagne d'une mobilisation à caractère ethnique. Encore limitée aux élites dans les milieux urbains au début de l'indépendance en 1962, la violence politique finit pourtant par embraser la campagne particulièrement en 1965, 1972, 1988, 1993 jusqu'en l'an 2000.

Ce recours récurrent à la violence politique finit par entamer la nation dans la mesure où elle se déroule sans que les acteurs tant internes qu'externes abordent les deux questions fondamentales auxquelles l'Etat et la société étaient en réalité confrontées. En effet, comment fonder la légitimité du pouvoir entre les deux principaux groupes (structure) et en même temps arrêter des mesures politiques et juridiques en vue de bannir le recours à la violence politique (processus) ? C'est en principe sur cette nouvelle base que le pouvoir consolidé pouvait traiter les autres questions structurelles et conjoncturelles de développement auxquels le pays reste fondamentalement confronté ${ }^{7}$. Il faudra attendre la guerre civile de 1993 pour que le pays, aidé par la communauté régionale et internationale, retrouve le chemin de la médiation et de la recherche du compromis lors de négociations de paix d'Arusha de juin 1998 à août 2000.

\section{2) Négociations et partage du pouvoir}

L'Accord d'Arusha pour la Paix et la Réconciliation au Burundi signé le 20 août 2000 à Arusha (Tanzanie) constitue un tournant historique dans le traitement des rivalités politicoethniques internes au Burundi. Certes, le pays est plongé dans une guerre civile depuis le coup d'état du 21 octobre 1993 où suite à l'assassinat du président Melchior Ndadaye, différents groupes rebelles hutu entrent en conflit armé contre l'Etat et les troupes gouvernementales à majorité tutsi. Cependant, des espaces de dialogue existent.

De 1993 à 1996, toutes les tentatives de ramener la paix et la sécurité dans le pays ont échoué ${ }^{8}$ tandis que la guerre civile continue. Il faudra attendre le retour au pouvoir du président Pierre Buyoya en juillet 1996 pour que la région et la communauté internationale s'emploient à trouver, à travers un dialogue plus ou moins inclusif, une solution jugée définitive à la crise burundaise. Après deux années de négociations (1998 à 2000), les parties burundaises, à savoir le gouvernement en place, des partis politiques, l'opposition non armée concluent ledit accord sous le parrainage de la communauté régionale et internationale. Dans cet ordre d'idées, l'Accord avait le mérite de cadrer, de définir le conflit burundais, à savoir « un conflit

\footnotetext{
${ }^{5}$ Gahama Joseph et. alii. : Op. Cit, p. 87

${ }^{6}$ Octobre 1965, Novembre 1966, novembre 1976, septembre 1987, juillet et octobre 1993, juillet 1996, mai 2015

7 Pour les questions structurelles et conjoncturelles de la Région des Grands Lacs (Rwanda et Burundi particulièrement), le lecteur peut consulter un article original de Griggs Richard A. (1997) : Geostrategies in the Great Lakes Conflict and spacial design for Peace ; in www.cwis.org visité le 12 mars 2016.

${ }^{8}$ Remise en scelle de l'Assemblée Nationale, élection d'un chef d'état, recentrage des questions politiques et sécuritaires dans les institutions étatiques et non dans la rue, l'Accord Kigobe signé le 04 février 1994
} 
fondamentalement politique avec des dimensions ethniques extrêmement importantes; un conflit découlant d'une lutte de la classe politique pour accéder au pouvoir et/ou s'y maintenir $»^{9}$. Ce recadrage, si l'on peut ainsi dire, permet à toutes les parties de s'y reconnaître. Il allie la dimension ethnique et politique du conflit, deux facteurs dont les esquisses d'une solution durable devraient tenir compte. Cette définition met particulièrement fin au phénomène de «méta-conflit ${ }^{10}$ consécutif au processus d'escalade de tout conflit durable.

Les mesures de politique générale que préconise l'Accord d'Arusha sont l'instauration d'un nouvel ordre politique, économique, social, culturel et judiciaire au Burundi, une nouvelle organisation structurelle des institutions de l'Etat, la mise en place d'institutions de transition en vue de garantir l'application de l'Accord, l'orientation des programmes des partis politiques vers des idéaux d'unité et de réconciliation nationale, la consécration du principe de la séparation des pouvoirs, la promulgation d'une loi électorale prenant en compte les préoccupations et les intérêts de toutes les composantes de la nation burundaise et, enfin, la prévention des coups d'Etat ${ }^{11}$.

Plus concrètement, l'Accord d'Arusha préconise un modèle de partage du pouvoir, "powersharing ", qui s'inspire du «consociationalisme», de la protection et de la promotion des droits humains. Ce modèle consiste à créer des conditions pour une solution constructive à un long conflit à travers la reconnaissance de l'appartenance «ethnique» des membres de l'élite, leur coopération en vue de résoudre les problèmes collectifs et ainsi éviter la création d'une "société segmentée " ${ }^{12}$. La Constitution de 2005 qui s'inspire dudit Accord définit les institutions fondamentales de l'Etat, accepte le principe démocratique tout en le tempérant pour que le conflit en apparence «ethnique » soit résolu en tenant compte des autres clivages locaux et régionaux : à titre d'exemple, deux vice-présidents d'obédience ethnique et politique différente, $60 \%$ et $40 \%$ des membres hutu et tutsi au sein du gouvernement, $50 \%$ de l'armée pour chaque groupe, au niveau national un parti ne peut pas être représenté à plus de $67 \%$ etc. à l'issue des élections locales ${ }^{13}$.

Le mérite de l'Accord d'Arusha aura sans doute été d'aller au-delà d'une simple restructuration de l'Etat. Il s'adresse de surcroît aux questions structurelles fondamentales du pays, celles sur lesquelles les partis n'ont pas d'emprise, mais auxquelles l'exercice du pouvoir doit s'attaquer en vue de répondre aux problèmes collectifs: questions d'éducation, de santé, d'infrastructures, la bonne gouvernance, la finalité d'un pouvoir politique, le développement économique, de croissance démographique. Autant de questions auxquelles le pays est confronté au-delà de l'appartenance à un groupe de sa population ${ }^{14}$.

Bref, les rédacteurs de l'Accord d'Arusha de 2000 et de la Constitution de mars 2005 qui en découle ont cherché à répondre et à résoudre deux questions fondamentales du conflit interne burundais depuis son indépendance, à savoir, sur le plan structurel, la légitimité du pouvoir et quant au processus, le bannissement du recours à la violence politique à travers l'esquisse des institutions et le choix de leur personnel. Conclu en l'an 2000, l'Accord s'inspire en réalité des standards juridiques internationaux consécutifs à la victoire du bloc libéral occidental qui prescrit les voies, les fondements de règlement des conflits internes, à savoir, les principes

\footnotetext{
${ }^{9}$ Article 4 alinéa a et $b$ de l'Accord

${ }^{10}$ Conflit sur le conflit ou sur la nature du conflit, et par conséquent, conflit sur ses voies de solutions possibles.

${ }^{11}$ Accord d'Arusha, article 5

12 Lustick Ian (1979): "Stability in deeply divided societies: Consociationalism versus Control"; in World politics, Vol. 31, Nr.3, pp. 325-344; voir Lijphard Arend (1977): Democracy in plural societies. A comparative exploration, Yale: Yale University Press.

${ }^{13}$ Constitution du 20 mars 2005, articles 129, 143, 164, 266

${ }^{14}$ Accord d'Arusha, Protocole IV : Reconstruction et développement
} 
démocratiques, le respect des droits de l'homme, une justice fonctionnelle, la coopération économique et, enfin, la satisfaction des besoins humains et collectifs fondamentaux ${ }^{15}$.

Naturellement, un accord, une constitution ne sont que des instruments qui déterminent les moyens politiques et légaux dont les membres d'une société politique disposent pour accéder, se maintenir au pouvoir, sinon jouir, en tant que citoyens ordinaires, des droits et des devoirs. L'accord ne pouvait certes pas résoudre tous les problèmes, mais il constituait seulement un début de réponse aux quatre décennies de crises; comme le dit Van Eck, «the Arusha Accord did not produce the 'promised land', at the best it has produced a roadmap showing how to get there» ${ }^{16}$.

Concrètement, une partie de l'opposition armée, deux groupes rebelles, le Conseil National pour la Défense de la Démocratie-Forces de Défense de la Démocratie (CNDD-FDD aujourd'hui au pouvoir) et le mouvement PALIPEHUTU- Front National de Libération devenu FNL comme parti politique, n'étaient pas parties à cet accord ayant pour ambition de traiter les termes du conflit dans toutes ses dimensions. Une fois l'application de l'Accord devenu irréversible et que le processus démocratique ait été déclenché, les deux parties restantes intégreront progressivement les institutions étatiques par le biais de la conclusion d'accords spécifiques ${ }^{17}$.

\section{3) Un seul cadre politique et légal, deux interprétations différentes}

Des élections générales ont eu lieu après la période de transition : la première législature de 2005 à 2010, la deuxième de 2010 à 2015. D'après un certain nombre d'analystes, la crise actuelle serait consécutive à la volonté du chef de l'Etat de se porter candidat pour un deuxième ou un troisième mandat ; comment alors peut-on alors expliquer cette crise ?

L'enjeu de l'Accord d'Arusha était de concevoir un modèle étatique qui conviendrait le mieux au Burundi au sortir des décennies d'instabilité politique et de guerre civile, et au regard de la situation du Burundi en l'an 2000; quinze ans après, la crise actuelle connaît donc une dimension politique et légale.

Le Protocole II relatif à la démocratie et à la bonne gouvernance stipule que «les parties audit accord sont convenues de veiller à ce qu'il soit élaboré pendant la période de transition, un texte constitutionnel pour le peuple burundais qui soit conforme aux principes énoncés dans le chapitre premier du présent protocole $(\ldots) \gg^{18}$. A la lumière de cette disposition, il est donc recommandé à la constitution post-transition «de stipuler qu'à l'exception de la toute première élection présidentielle, le Président de la République est élu au suffrage universel direct, chaque électeur ne pouvant voter que pour un seul candidat. Le Président de la République est élu à la majorité absolue des suffrages exprimés (...) ${ }^{19}$. Cette disposition sera reprise plus loin, le premier Président de la période post-transition étant élu par l'Assemblée nationale et le Sénat réunis, à la majorité des deux tiers ${ }^{20}$. En outre, «le Président de la République est élu pour un mandat de cinq ans renouvelable une seule fois.

\footnotetext{
${ }^{15}$ Wallensteen Peter (2012): Understanding conflict resolution. War, Peace and the global system, $3^{\text {rd }}$ edition, London: Sage publications, pp.10-11

${ }^{16}$ Van Eck Jan (2001): “Polarization of parties into 'win power' and 'keep power' camps, threatens the collapse of peace process"; in Burundi Report Nr. 2001/1, p.1

17 Après l'Accord d'Arusha, huit accords distincts ont été signés soit par le Gouvernement de transition (novembre 2001 à mai 2005) soit par le gouvernement issu des élections de 2005 avec le groupe rebelle restant, respectivement le CNDD-FDD et le FNL

${ }^{18}$ Accord d'Arusha, Protocole II : Démocratie et Gouvernance, préambule.

${ }^{19}$ Accord d'Arusha, Protocole II : Démocratie et Gouvernance, article 7 alinéa 1 a)

${ }^{20}$ Accord d'Arusha, Protocole II : Démocratie et Gouvernance, article 20, alinéa 10
} 
Nul ne peut exercer plus de deux mandats présidentiels ${ }^{21}$. Traitée de façon redondante dans le Préambule II de l'Accord d'Arusha, la question du mandat présidentiel est pourtant abordée de façon sommaire dans le texte légal qui s'en inspire.

La Constitution du Burundi stipule que «le Président de la République est élu au suffrage universel direct pour un mandat de cinq ans renouvelable une fois $»$, donc dix ans. ${ }^{22}$ Le Titre $\mathrm{XV}$ met sur pied des dispositions particulières pour la première période post-transition. Ainsi, «à titre exceptionnel, le premier Président de la République de la période post-transition est élu par l'Assemblée Nationale et le Sénat élus réunis en Congrès, à la majorité des deux tiers des membres (... $)^{23}$.

La question de la fin du mandat présidentiel et de l'alternance du pouvoir soulève des attentes démesurées et des mises en question. Pour le gouvernement et ses partisans, seul le cadre légal compte, à savoir l'article 96 de la constitution qui traite le nombre de mandats présidentiels, 10 ans au maximum, et le mode d'élection, une élection au suffrage universel direct. Autrement dit, l'exception soulevée par l'article 302, à savoir une élection au suffrage indirect et à titre exceptionnel pour le premier Président après la transition, n'est pas à tenir en considération. Pour l'opposition, l'article 96 s'avère certes d'application, mais avec des nuances. L'article 302 porte sur le mode d'élection à titre exceptionnel pour l'élection du premier Président de la République post-transition, et non sur le nombre des mandats du Président. En outre, les dispositions de l'Accord d'Arusha prévalent dans la mesure où elles limitent les mandats possibles à dix ans au maximum. C'est sur fonds d'interprétations divergentes des mêmes textes que le délai de présentation des candidatures aux différentes élections en vue approchait. Il était donc urgent que le Chef de l'Etat tranche la question de sa candidature.

\section{4) D'une différence d'interprétations à une crise institutionnelle}

Un rapport, en principe confidentiel, du Chef du Service National du Renseignement (SNR) de l'époque est rendu public en février $2015^{24}$. Ce dernier suggère que le troisième mandat devrait tenir compte non seulement du prescrit de la constitution, mais aussi des opinions des autres partenaires du Burundi, entre autres, les confessions religieuses, les puissances régionales et internationales lesquelles déconseillent au président de rempiler pour un troisième mandat. En outre, il expose les dangers que court le pays en cas d'une candidature de l'actuel Chef de l'Etat à un troisième mandat. Dans le même ordre d'idées, dix-sept membres du parti au pouvoir dont le Porte-parole du Président de la République, le Porteparole du parti au pouvoir CNDD-FDD et d'autres cosignent le 20 mars 2015 un écrit adressé au Chef de l'Etat dans lequel ils entendent faire part de leur position par rapport à la candidature au troisième mandat.

Dans les deux cas, le responsable des services de renseignement de cette époque, le Général Godefroid NIYOMBARE, sera limogé à la suite de la publication dudit rapport ${ }^{25}$ d'une part, et d'autre part, les signataires de ladite lettre seront démis de leurs fonctions politiques et administratives. Ces deux décisions prouvent à suffisance que le Chef d'Etat avait déjà pris la décision de se porter candidat à un nouveau mandat.

\footnotetext{
${ }^{21}$ Accord d'Arusha, Protocole II : Démocratie et Gouvernance, article 7, alinéa 3

${ }^{22}$ Constitution du Burundi, article 96

${ }^{23}$ Constitution du Burundi, Article 302

${ }^{24}$ Note de renseignement: L'éventuelle candidature du Président Nkurunziza au troisième mandat est-elle constitutionnelle? Positions des différents partenaires et orientations du SNR, Fiabilité 96\%, Bujumbura, le 13 février 2015

${ }^{25}$ Lequel conduira une tentative de coup d'état avorté le 13 mai 2015
} 
Lors d'un congrès du parti au pouvoir, le CNDD-FDD, tenu le samedi 25 avril 2015, le Chef de l'Etat annonce publiquement sa décision de se porter candidat pour un troisième mandat. Aussitôt, des manifestations contre cette décision débutent dans certains quartiers de la capitale Bujumbura et à l'intérieur du pays, tandis que d'autres dénoncent ou soutiennent la décision à travers des médias sociaux en fonction de leur appartenance politique.

Malgré cette déclaration, et au regard de la tournure inattendue que prend le cours des événements, 14 sénateurs du parti au pouvoir, le CNDD-FDD, introduisent une lettre de requête à la Cour Constitutionnelle demandant aux juges d'interpréter les articles controversés de la Constitution (articles 96 et 302). Ces derniers décident d'un «mandat tout à fait exceptionnel et spécial» autorisant ainsi le Président sortant à briguer un troisième mandat ${ }^{26}$.

\section{5) Une instabilité politique aux conséquences internes}

Au-delà des mouvements de soutien ou des soulèvements de désapprobation, la crise produit ses conséquences d'abord sur le plan humanitaire. Il s'observe un flux de réfugiés en direction des pays limitrophes. D'après le Haut-Commissariat des Nations Unies pour les Réfugiés (HCR), il a été enregistré depuis avril 2015, 250.473 personnes en qualité de réfugiés en République Démocratique du Congo (21.186), au Rwanda (73.926 dont environ 25.000 dans la ville de Kigali), en Tanzanie (131.834), en Ouganda (22.330) et en Zambie (1.197) et dans d'autres pays ${ }^{27}$. Sous le même registre, notons que le Commissariat belge pour les Réfugiés et Apatrides (CRA) a enregistré 364 demandeurs d'asile burundais, un nombre qu'on n'avait pas atteint pendant la longue guerre civile des années 90 malgré les difficultés actuelles d'octroi $\mathrm{du}$ visa d'entrée et du permis de séjour ${ }^{28}$. Cette conséquence humanitaire va encore s'amplifier puisque le HCR fait état de nouveaux arrivants par semaine de l'ordre de 1000 en Tanzanie, 500 en Ouganda, 230 au Rwanda et 200 en République Démocratique du Congo en dépit de quelques retours spontanés qui ont déjà eu lieu. La répression des manifestations dites anti-troisième mandat aurait occasionné 400 morts, un bilan qui varie en fonction des sources. La plupart de ces victimes, forces de l'ordre et manifestants compris, appartiennent aux deux groupes et à une génération encore jeune au moment de la guerre civile des années quatre-vingt-dix. Elles consomment les médias sociaux et surtout vivent des conditions précaires dans les milieux urbains et ruraux ${ }^{29}$.

Sur le plan politique, des effets négatifs se font remarquer. Le processus électoral constitue malheureusement, 25 ans après le processus de démocratisation en Afrique, une source majeure de crises. Il s'agit d'un contraste dans la mesure où des élections tenues particulièrement après une législature ont pour mérite en principe de consolider la démocratie et de renouveler les programmes de gouvernement par de nouvelles idées. La reconstruction politique en cours était le fruit d'âpres négociations ayant conduit à l'Accord d'Arusha pour la paix et la Réconciliation au Burundi, quatre années de transition et l'organisation d'élections générales en 2005, 2010 et 2015. Par rapport aux décennies antérieures, le pays constituait un Etat moderne avec les requis d'un système politique ayant pris en compte les dimensions historiques de l'instabilité politique interne (depuis l'accession du Burundi à l'indépendance) et la volonté d'inclure toutes les composantes de la population à travers les institutions, un

\footnotetext{
${ }^{26}$ Voir à ce propos le journal IWACU N³39 du 11.09.2015 et Vandegiste Stef (2014) : La limitation constitutionnelle du nombre de mandats présidentiels: Une coquille vide? Une analyse du cas du Burundi, Working paper 2014/04, University of Antwerp/IOB

${ }^{27}$ Communiqué du HCR cité par l'agence de presse belge Belga du 05 mars 2016

${ }^{28}$ D'après le Rapport mensuel de janvier 2016, Statistiques d'asile et des rapports antérieurs, il y avait en 2000 305 demandeurs d'asile en Belgique, en 2016364 et en 2001255

${ }^{29}$ Voir Guichaoua André : «Le refus du troisième mandat ne suffit pas à expliquer l'ampleur des protestations»; in RFI du 11.06.2015
} 
système de quota que le pays devrait en principe se défaire avec le temps, une fois la gestion de l'Etat assainie et la réconciliation atteinte.

Les mouvements de protestation contre le troisième mandat sont également le fruit de frustrations de ces dernières décennies: Une démographie galopante qui annihile la croissance économique, des secteurs de production économique encore au niveau embryonnaire etc. Cet ensemble de facteurs a pour effet que chaque année des milliers de jeunes scolarisés et des jeunes ruraux en quête d'emploi viennent grossir les villes et les centres urbains secondaires à travers le pays. Lors de la présentation du budget 2015-2016, le Ministre des Finances et de la Planification rend public l'ampleur du chômage au Burundi : $18,9 \%$ de la population ayant le niveau du secondaire général et $12 \%$ de Burundais possédant le niveau d'enseignement supérieur sont sans emploi ${ }^{30}$.

Au-delà des conséquences internes, cette crise nous rappelle encore une fois que les difficultés internes à un Etat se répercutent également sur les autres pays de la région et de façon négative ; ces conséquences pèsent également sur ses relations avec les pays limitrophes et certains partenaires au niveau international.

\section{6) Des répercussions au niveau régional et international}

La crise politique actuelle se répercute négativement sur les relations du pays avec l'extérieur. Dans un premier temps, le Burundi entretient aujourd'hui de mauvaises relations avec le Rwanda. L'instabilité interne aux deux pays a provoqué des flux de réfugiés dans les pays limitrophes au point que des groupes hostiles, voire rebelles à un Etat élisent domicile dans un autre. Depuis le génocide rwandais de 1994 existent en République Démocratique du Congo (RD Congo) les «Forces Démocratiques pour la Libération du Rwanda » (FDLR) accusées par Kigali d'avoir participé au génocide et de chercher à le combattre. Le régime rwandais accuse le Burundi de collaborer, de loger des membres de ce groupe sur son territoire. De son côté, le Burundi accuse le Rwanda d'héberger des réfugiés dont une partie serait entraînée au maniement des armes et au combat militaire par le Rwanda en vue de le déstabiliser ${ }^{31}$.

En suivant au quotidien ce développement négatif, il n'a jamais été question entre les deux pays de s'engager dans un dialogue ouvert et inclusif sur le plan diplomatique relatif aux termes du conflit, dans lequel évidemment la question de sécurité pour les deux pouvoirs en place est en réalité posée. Ce sont deux pays membres de 1' «East African Community» (EAC), de la Communauté Economique des Pays des Grands Lacs (CEPGL), de la «Common Market of Eastern and Southern Africa»(COMESA), de la «Communauté Internationale pour la Région des Grands Lacs» (CIRGL) constituant, entre autres, de forum de dialogue y compris pour des questions vitales de sécurité. La situation des Etats de la sous-région est intrinsèquement interdépendante au point qu'une crise dans un pays connaît des répercussions négatives sur l'ensemble de la région.

Au-delà des frictions dans les relations actuelles entre le Rwanda et le Burundi, c'est la nature du rôle joué par la présence des refugiés sur le territoire d'un autre pays qui est mise en exergue. Cette présence contribue, sur le plan théorique et empirique, à fixer dans le pays d'origine les facteurs politiques, économiques et autres qui l'ont créée et constituer une cause de mauvais voisinage entre pays limitrophes ${ }^{32}$. Dans le cas sous revue, certains auteurs esquissent l'éventualité d'un scénario catastrophique pour la région. En effet, si une crise ouverte voire une guerre éclatait entre le Rwanda et le Burundi, force est de se demander si la

\footnotetext{
${ }^{30}$ www.arib.info visité le 10 mars 2016

${ }^{31}$ Rapport des Nations Unies: Letter dated 15 january 2016 from the coordinator of the Group of Experts on the DRC adressed to the Chair of the Committee on DRC (UN Resolution 1533/2004); Les mythes et les réalités d'une "guéguerre" entre Bujumbura et Kigali, PANA 14.02.2016

32 "Hosting and Sending States».
} 
région resterait neutre ou plutôt des pays comme la Tanzanie, la RD Congo, l'Angola n'entreraient pas en jeu pour soutenir l'une des parties en conflit et, ainsi, voir se dessiner un conflit régional aux conséquences imprévisibles ${ }^{33}$. Les parties étant averties sur cette éventualité, ne serait-ce que dans les divers articles publiés actuellement dans les médias sociaux, des menaces existent sur d'autres fronts régionaux.

Bref, au-delà des relations bilatérales, la Communauté Est-Africaine et 1'Union Africaine paraissent incapables de résoudre une crise interne ou opposant deux pays membres. Des études devraient être conduites à ce sujet au-delà des contraintes en matière de ressources personnelles et le manque d'infrastructures technologiques que connait la section de 1' «African Union's Continental Early Warning System (CEWS)» en matière de collecte de données et d'échange d'informations de façon efficiente entre les cinq communautés économiques régionales (REC's) reconnues par l'Union Africaine ${ }^{34}$.

Pour certains analystes, la crise actuelle met en cause la participation du Burundi à des missions de maintien de la paix pour lesquelles les troupes burundaises étaient appréciées : environ 2500 soldats en Somalie dans le cadre de l'AMISOM, 850 soldats en République Centrafricaine (MINUSMA), et d'autres en Côte d'Ivoire, en Haïti, au Darfour, etc. La participation de ces troupes à ces différentes missions constitue non seulement une source de prestige pour le Burundi à cause de leur efficacité sur le terrain, mais également une source importante de revenus tant pour les individus concernés que pour l'Etat qui en retire des devises à divers titres tels que les revenus versés, les frais d'entretien des équipements, les missions d'évaluation, etc.

Sur le plan économique, le pays connaît une régression sans précédent en une période supposée de retour à la paix. Le Burundi dépend de l'aide extérieure en vue de financer son développement, et les partenaires importants du Burundi ont suspendu leur aide. Les USA ont exclu le Burundi de 1' "Act of Growth Opportunity Act» (AGOA) depuis le début de cette année 2016, suivis par la Belgique, l'Allemagne, la Hollande, la France, qui ont, à leur tour, gelé l'aide accordée au Burundi. L'Union Européenne, premier bailleur de fonds du Burundi (430 millions d'euros pour la période 2015-2020), vient de suspendre ses appuis financiers directs fournis à l'administration burundaise, y compris les appuis budgétaires $(52 \% \mathrm{du}$ budget), tout en affirmant maintenir intégralement son soutien financier à la population et son aide humanitaire ${ }^{35}$. L'effet conjugué de toutes ces mesures est la dévaluation subséquente de la monnaie, l'inflation suite au manque de production $^{36}$, la cherté de la vie, la fermeture d'entreprises et un chômage de masse qui perdure.

En outre, la crise en cours au Burundi remet en cause les programmes et projets de consolidation de la paix pour lesquels le Burundi et la Sierra Léone avaient été élus en décembre 2006. Ces derniers ont été financés par un Programme de Consolidation de la Paix ou «Peace Building Funds» (PBF) doté de 34 millions US\$. Après les opérations de maintien de la paix au Burundi (ONUB, 2004-2006), le Conseil de Sécurité a mis sur pied le Bureau Intégré des Nations Unies au Burundi (BINUB, 2006-2009) avec pour mandat de «continuer d'aider le Gouvernement burundais à consolider la paix en renforçant les capacités nationales nécessaires pour s'attaquer aux causes profondes du conflit $\gg^{37}$.

\footnotetext{
${ }^{33}$ Lire Reyntjens Filip et la crise burundaise in www.blog.lesoir.be visité le 20 mars 2016 ; interview dans Iwacu du 13 novembre 2015

${ }^{34}$ Voir Interview du Chef de la Division "AU's Early Warning Division" in Institute for Security Studies (University of Pretoria) Press Release du 25 avril 2016: "Linking early warning to early responses".

${ }^{35}$ Burundi : L'UE clôture les consultations au titre de l'article 96 de l'Accord de Cotonou, Communiqué de presse 115/16 du lundi 14 mars 2016

${ }^{36}$ Le FMI prévoit une diminution de 7,2\% du Produit Intérieur Brut (PIB)

${ }^{37}$ Résolution 1719 du Conseil de Sécurité du 25 octobre 2006 recommandant la mise sur pied du BINUB
} 
En effet, l'Agenda pour la Paix publié en 1992 par l'ancien Secrétaire Général des Nations Unies, Boutros Boutros-Ghali discute, entre autres, des activités à mener avant, pendant et après un conflit. Quoique les idées prônées ne fussent pas neuves, son originalité aura été de les rassembler dans un cadre politique et ainsi d'offrir une compréhension commune et cohérente des instruments disponibles en vue de résoudre un conflit comme il y en avait beaucoup au sortir de la guerre froide ${ }^{38}$. La consolidation de la paix s'entend comme un ensemble d'actions visant à identifier et soutenir des structures qui tendent à renforcer la paix en vue d'éviter à une société et à un Etat de retomber dans un conflit. Loin d'un ensemble d'actions à conduire après qu'un conflit ait pris fin, il s'agit aujourd'hui plutôt de reconnaître que la consolidation de la paix constitue un processus à long terme et ainsi concevoir une approche globale pour soutenir la paix. Et dans cet ordre d'idées, l'ONU a mis sur pied une architecture de consolidation de la paix, l'Union Africaine a créé un cadre pour les opérations de maintien de la paix et mis sur pied un cadre de reconstruction et de développement postconflit.

Le problème posé est que les praticiens de la consolidation de la paix sur terrain travaillent sous la pression de montrer des résultats palpables pour lesquels ils sont comptables vis-à-vis des bailleurs («accountability»), de la communauté internationale et de justifier, sinon de montrer, des réalisations concrètes. La conséquence de cette pression conjuguée est de se concentrer plutôt sur des réponses à court terme dont on n'est pas si sûr qu'elles répondent réellement aux causes des conflits.

En vue de pallier à ce déficit, l'ONU revoie son actuelle architecture de consolidation de la paix. L'Institute of Security Studies (ISS) de l'Université de Pretoria (Afrique du Sud) conduit depuis une année des études de ce genre en vue de concevoir des programmes et projets de consolidation de la paix fondés sur des faits avérés sur terrain, tandis que des groupes de recherche ont déjà mené des réflexions à cette fin ${ }^{39}$.

\section{7) Des tentatives unilatérales de résolution de la crise}

Différents acteurs ont tenté de résoudre sans succès apparent la crise en cours. Au-delà des protestations, une tentative de coup d'Etat du 13 mai 2015 a échoué. Dans l'étude des conflits, un coup d'état est un instrument également illégal et constitue une guerre civile de faible intensité dans la mesure où un groupe cherche à saisir le pouvoir en chassant un autre. Les auteurs avaient annoncé la dissolution du gouvernement et la mise en place d'un comité de transition «pour le rétablissement de la concorde nationale, ayant pour mission, entre autres, le rétablissement de l'unité nationale ${ }^{40}$.

Cette tentative de coup de force a connu un échec car elle n'a pu rallier qu'une petite frange des forces de sécurité. Son échec a eu pour effet de consolider plutôt le régime de Bujumbura appuyé, entre autres, par l'armée et la police, en affaiblissant tous les porteurs de voix alternatives dans le pays qui sont accusés d'être du côté des putschistes à savoir des membres de la société civile, des journalistes et opposants aujourd'hui en exil. Cette thèse est défendue par Laurent Kavakure, ancien ministre des Relations extérieures à l'époque du coup d'Etat, dans son récent ouvrage. Pour lui, «les auteurs de violence voulaient renverser le pouvoir en

\footnotetext{
${ }^{38}$ De Carvalho Gustavo, Kok Onnie (2016): Does anyone know what good peace building looks like? ISS Presse release du 24 février 2016; voir Hamdi Mehdi (2009): Les opérations de consolidation de la paix, thèse de doctorat, Université d'Angers.

${ }^{39}$ Geneva Peacebuilding Platform (2015): White Paper on Peacebuilding, Geneve; Friederich Ebert Stiftung / FriEnt (2014): A New Deal for the Peacebuilding Commission? Expert Discussion, 03 November 2014. UNICEF Burundi et l'Université d'Amsterdam conduisent actuellement une étude sur "La transmission intergénérationnelle de la violence et la résilience au Burundi".

${ }^{40}$ Le Monde du 13 mai 2015
} 
place incarné par le CNDD-FDD, et mettre au rancart la démocratie burundaise si chèrement négociée à Arusha (...). L'opération avait été longuement murie et planifiée avec une bonne logistique et un système de communication performant ${ }^{41}$.

C'est dans ce contexte que des élections générales ont été conduites permettant au parti au pouvoir de gagner les élections ${ }^{42}$, d'affermir davantage son emprise sur l'Etat et l'administration publique et de mettre fin à la discussion relative au troisième mandat. Il reste néanmoins à gagner la confiance de la communauté internationale, particulièrement les bailleurs bi- et multilatéraux. L'Union Européenne vient de geler son aide au Burundi dans le cadre de l'Accord de Cotonou (Bénin). Cela prive le Burundi de son appui à la consolidation de l'Etat de 143 millions d'euros dont une aide budgétaire directe de $52 \%$ du Budget et des appuis sectoriels dans des domaines tels que la justice, les finances publiques, le programme de décentralisation, des infrastructures, etc. ${ }^{43}$

Des tentatives de sortie de crise ont été initiées sous la conduite de médiateurs des Nations Unies et du Président Yoweri Museveni mandaté par la Communauté Est-Africaine, sans grand succès, tandis qu'un sommet des Chefs d'Etat de la Communauté Est-Africaine vient de nommer l'ancien président tanzanien Benjamin MKAPA comme médiateur aux côtés du président ougandais.

L'Union Africaine de son côté à travers son Conseil de Paix et Sécurité avait proposé le déploiement d'une Mission Africaine de Prévention et de Protection au Burundi (MAPROBU) composé de 5000 personnels militaires et de police. Son mandat était, entre autres, de «prévenir toute détérioration de la situation sécuritaire, d'en surveiller l'évolution et de rendre compte de la situation sécuritaire, d'en surveiller l'évolution et de rendre compte des développements intervenant sur le terrain (...) $»^{44}$. Cette proposition fut rejetée par le gouvernement du Burundi alléguant la souveraineté du pays et menaçant de combattre cette «force d'invasion ». Face à ce refus, l'ONU a suggéré l'envoi plutôt d'une équipe d'observateurs des droits de l'homme et de politiciens non armés.

\section{8) Dialogue et sens du compromis pour sortir de la crise}

Les conséquences de la crise en cours étant multidimensionnelles, les acteurs internes, épaulés par des médiateurs régionaux et internationaux, travailleraient plutôt à la recherche d'un compromis, en vue d'aboutir à une solution dans laquelle les parties primaires, le pays et la région se trouveraient tous bénéficiaires.

Sur le plan structurel, un dialogue inclusif sur les termes réels du conflit et les voies de solution alternatives rassemblant tous les acteurs, sous la houlette de médiateurs nationaux et/ou étrangers reste incontournable. L'actuel dialogue politique interne devrait faire participer tous les acteurs à l'intérieur comme à l'extérieur du pays en vue d'aboutir à un compromis inclusif et en diffuser les résultats obtenus à l'ensemble de tous les secteurs de la vie nationale.

Sur le plan conjoncturel, trois questions intrinsèquement liées méritent une attention particulière. Il s'agit du troisième mandat du Chef d'Etat en exercice, la problématique de la bonne gouvernance, et l'enjeu de la violence politique et l'impunité. Des concessions mutuelles autour de ces trois questions restent nécessaires en vue de sortir durablement de la

\footnotetext{
${ }^{41}$ Kavakure Laurent (2016) : Burundi. La démocratie à l'épreuve (Août 2010-août 2015), Bujumbura : Presses Lavigerie, p.10.

4220 juin 2015, Elections de Députés et des Conseillers communaux ; 21 juillet, Election présidentielle ; 24 juillet, élections des Sénateurs, 24 août, élections de conseillers collinaires.

43 Voir également «Burundi: La paix sacrifiée?» International Crisis Group Briefing Afrique $N^{\circ} 11$, Nairobi/Bruxelles du 29 mai 2015.

${ }^{44}$ Communiqué du Conseil de Paix et de Sécurité de l'Union Africaine, 565 ${ }^{\text {ème }}$ réunion du 17 décembre 2015.
} 
crise. Pour cela, les parties devraient être animées d'un esprit constructif en vue d'une solution «gagnant-gagnant».

Il sied, à cet effet, d'apprécier le pas déjà franchi par le pays, autrement dit, les développements récents de la vie politique interne par rapport aux décennies précédentes caractérisés par un processus démocratique qui était l'un des plus avancés dans la sous-région. Citons, entre autres, la conclusion de l'Accord d'Arusha en tant que méthode et résultats (médiation, dialogue et négociations), l'adoption de la Constitution, la période de transition ayant marqué le début d'une appropriation de l'accord conclu par une partie de l'élite à Arusha, les élections générales, la liberté d'expression déjà acquise, des rapports politicoethniques moins tendus. Ce sont des atouts que le pays a progressivement acquis au cours de ces quinze dernières années et qui ont pu empêcher toute mobilisation d'ordre politicoethnique à grande échelle.

\section{Conclusion}

La présente contribution constitue une recherche exploratoire sur un sujet d'actualité en évolution et susceptible d'évoluer encore au cours des jours et mois à venir. La question de délimitation des mandats présidentiels suscite dans la Région des Grands Lacs (Burundi et République Démocratique du Congo, Congo-Brazzaville, Rwanda) des questionnements sur le système démocratique souhaité conduisant parfois à des crises internes.

Chaque cas étant particulier, il a été question d'abord de nous concentrer de façon analytique sur le cas du Burundi qui, depuis fin avril 2015, est entré dans une crise malgré des développements politiques récents depuis la conclusion de l'Accord d'Arusha en août 2000. Cet Accord, suivi de deux périodes de transition et des élections générales, avait contribué à répondre à deux questions fondamentales à savoir la légitimité du pouvoir et le bannissement de la violence politique. Le dialogue politique et les négociations inclusives tout en acceptant des concessions réciproques restent les voies les meilleures pour sortir définitivement de la crise, en vue de s'attaquer aux problèmes du pays.

\section{Références}

Accord d'Arusha pour la Paix et la Réconciliation au Burundi, Août 2000

Campbell P. Susanna, Kayobera Léonard et Nkurunziza Justine (2010) : Evaluation indépendante externe. Projets du Fonds de consolidation de la Paix au Burundi, Bujumbura.

Centre for Humanitarian Dialogue (2011): The African Union and the search for peace and reconciliation in Burundi and Comoros, Addis Ababa

De Carvalho Gustavo et Kok Onnie (2016): Does anyone know what good peace building looks like? ISS Presse release du 24 février 2016

Friederich Ebert Stiftung et FriEnt (2014): A New Deal for the Peace building Commission? Expert Discussion, Berlin 03 November 2014.

Gahama Joseph, Makoroka Stanislas et. alii (1999): Burundi; in Adedeji Adebayo (ed.): Comprehending and mastering African conflicts. The search for sustainable peace and good governance, London/New-York: Zed Books, pp.80-103

Geneva Peace building Platform (2015): White Paper on Peace building, Geneva

Griggs A. Richard (1997): Geostrategies in the Great Lakes Conflict and spacial design for Peace; in www.cwis.org

Hamdi Mehdi (2009): Les Opérations de consolidation de la paix, Thèse de doctorat, Université d'Angers

Isabirye B. Stephen et Kooros Mahmoudi (2000): Rwanda and Burundi: The dynamics of their "tribal conflicts"; in Africa Quarterly, Vol.40, Nr.4, pp. 21-50 
Kavakure Laurent (2016): Burundi. La démocratie à l'épreuve. Août 2010-Août 2015, Bujumbura: Presses Lavigerie

Loi $\mathrm{N}^{\circ} 1 / 010$ du 18 mars 2005 portant promulgation de la Constitution de la République du Burundi

Lijphard Arend (1977): Democracy in plural societies. A comparative exploration, Yale: Yale University Press.

Lustick Ian (1979): "Stability in deeply divided societies: Consociationalism versus Control"; in World politics, Vol. 31, Nr.3, pp. 325-344

Ndayisaba Leonidas (2011): Evaluation de l'effet des projets de paix et gouvernance réalisés par le PNUD au Burundi de 2005 à 2009. Mission d'évaluation indépendante, Bujumbura in : http://www.erc.undp/evaluation/documents/download/5238

-- (2015) : Konflikt und Konfliktlösung im Gebiet der Großen Seen Afrikas. Eine Studie über Konfliktursachen und Krisenmanagement in Ruanda und Burundi, Saarbrücken: Südwestdeutscher Verlag für Hochschulschriften

Rufyikiri Gervais (2016): «Corruption au Burundi: Problème d'action collective et défi majeur pour la gouvernance», Working paper 2016/07, University of Antwerp/IOB

Van Eck Jan (2001): 'Polarization of parties into 'win power' and 'keep power' camps, threatens the collapse of peace process", Burundi Report Nr. 2001/1, p.1

Vandegiste Stef (2014): «La limitation constitutionnelle du nombre de mandats présidentiels : Une coquille vide ? Une analyse du cas du Burundi », Working paper 2014/04, University of Antwerp/IOB

Wallensteen Peter (2012): Understanding conflict resolution. War, Peace and the global system, $3^{\text {rd }}$ edition, London: Sage publications 